\title{
Recreational Drugs: a New Health Hazard for Patients with Concomitant Chronic Liver Diseases
}

\author{
Giovanni Tarantino $^{1^{*}}$, Vincenzo Citro ${ }^{2}$, Carmine Finelli ${ }^{3}$
}

1) Department of Clinical

Medicine and Surgery,

Federico II University

Medical School of Naples;

2) Department of Internal

Medicine, Umberto I Hospital,

Nocera Inferiore, Salerno;

3) Center of Obesity and

Eating Disorders, Stella Maris

Mediterraneum Foundation,

C/da S. Lucia, Chiaromonte,

Potenza, Italy

*INT Fondazione Pascale -

Cancer Research Center of

Mercogliano

Mercogliano (Av), Italy

\author{
Address for correspondence: \\ Giovanni Tarantino, MD \\ Department of Clinical \\ Medicine and Surgery, \\ Federico II University \\ Medical School of Naples \\ Naples, Italy \\ tarantin@unina.it.
}

giovanni.tarantino5@me.com

Received: 28.12.2013

Accepted: 13.01.2014

\begin{abstract}
Our purpose in this article is to review the effects of recreational drugs, used either on their own but principally combined with alcohol consumption, in determining hepatic injury or influencing the evolution of some chronic diseases of the liver, specifically HCV infection and NAFLD. A deleterious role of daily use of recreational drugs, in particularly cannabis, has been shown to demonstrate clearly a rapid progression of fibrosis and steatosis, leading to a major severity in patients with chronic hepatitis C. On the other hand, the effects of the misuse of these substances on NAFLD, the main obesity-related comorbidity, leading to addiction, is still to be elucidated even though some clues to the recreational drugs hepatotoxicity are already present in the literature. This short review aims at raising awareness about this topic.
\end{abstract}

Key words: non-alcoholic fatty liver disease - alcohol - recreational drug - cannabis - HCV infection.

\section{INTRODUCTION}

As it is well known to every expert in the field of hepatology, HCV infection, with its burden of progression toward liver cirrhosis, has an intrinsic high risk of developing hepatocellular carcinoma, a leading cause of death in adult males. The incidence of this is expected to decrease with the availability on the market of new antiviral drugs.

While viral $\mathrm{C}$ hepatitis has been mainly approached by hepatologists for the past two decades, we will focus on the liver disease which is nowadays recognized as being the most common type of chronic liver disease in Western countries. The disproportionately high intake of calories, also the so-called "empty" ones from alcohol, has led to a new pandemia, i.e. obesity [1]. A high percentage of obese patients generally suffer from non-alcoholic fatty liver disease (NAFLD). The spectrum of NAFLD includes simple fatty liver, non-alcoholic steatohepatitis (NASH) and advanced liver disease, which leads to liver-related failure and finally hepatocellular carcinoma [2]. It has been associated with many obesity-related health complications, including cardiovascular disease, type 2 diabetes, hyperlipidemia, and hypertension. This constellation is also recognized as the metabolic syndrome and is characterized by underlying insulin resistance [3-14]. Experts advise that it is necessary to reduce visceral fat to improve survival, mainly taking into account the strict link between NAFLD and coronary artery disease [15], in order to increase longevity [16].

In fact, the development and progression of fatty liver is closely associated with obesity, and is also attributed to excessive alcohol intake $[17,18]$. It is noteworthy to stress that heavy consumption of alcohol, the usual habit of the week-end-consumers, has been established to be a high risk factor for the liver, but light to moderate consumption is associated with a reduced risk of a fatty liver, suggesting a dose dependent relationship between alcohol consumption and hepatic steatosis.

Recreational drugs use is the habit of consuming a particular psychoactive substance with the intention of creating or enhancing a recreational experience, greatly implemented by peer pressure. It is characterized by the continued, compulsive use of this type of drugs, or the repetition of such deleterious behavior despite adverse consequences, or a neurological impairment leading to such behaviors [19]. These behavioral changes develop gradually during repeated exposure to 
drug abuse, and can persist for months or even years after discontinuation [20], suggesting that this addiction can be considered a form of drug-induced neural plasticity [21-23]. Several studies have shown that addiction is a disease that can target very specific regions of the brain and thus, any epigenetic intervention must not only be specific for a particular segment of the DNA, but it will also need to be targeted to a specific brain region $[24,25]$. Thus, chronic drug exposure causes stable changes in the brain at the molecular and cellular levels that underlie these behavioral abnormalities [26]. Literature data on effects of recreational substances, apart from alcohol that is managed in a section below, on NAFLD are lacking, which is surprising considering that some of them are responsible for acute cytolytic hepatitis, showing an intrinsic hepatotoxicity. It should be highlighted that recreational substance abuse remains difficult to prove since taking the product is often denied by the users.

Our purpose in this mini-review is to put in evidence the possible liver injury due to recreational drugs, consumed on their own but more frequently combined with alcohol excess in patients already suffering from HCV infection or NAFLD or both.

\section{INJURY OR BENEFITS OF ALCOHOL IN NAFLD: A LONG-LASTING CONTROVERSY}

First of all, we should take a glimpse into the more common recreational drug that is alcohol, with a special approach to obese patients.

Most obese patients with NAFLD have simple or partially benign [2] hepatic steatosis in the absence of necroinflammatory features or fibrosis; however, a significant minority may have NASH, which is characterized by hepatocellular inflammation and fibrosis [27]. Prolonged duration of NASH may ultimately lead to cirrhosis, in many cases called "cryptogenic cirrhosis" [28]. As previously evidenced, obesityrelated insulin resistance is one of the main pathogenic factors responsible for this progression, as well as hyperinsulinemia has been shown to increase the levels of free fatty acids and cholesterol [29] within the liver, resulting in cytotoxicity due to oxidative stress, which then promotes hepatic stellate cell activation and fibrosis [30]. As in other chronic liver diseases, alcohol seems to be an important risk factor for progressive fibrosis in NASH. Gabele et al [31] showed that a new model, in mice, allows the investigation of isolated or joint effects of alcohol and high fat diet on hepatic injury, where alcohol and high fat diet appear to act synergistically on the development of hepatic fibrosis, potentially via enhanced Toll-like receptor 4 (TLR4) signaling, which is known to play a crucial role in hepatic fibrosis. Wang et al [32] also showed, in rats, that even moderate alcohol consumption can cause more hepatic inflammation and cellular apoptosis in a pre-existing NASH condition. Ekstedt et al [33] showed, in humans, that moderate alcohol consumption, consistent with the diagnosis of NAFLD to be set, is associated with fibrosis progression in NAFLD. These patients should be advised to refrain from heavy, even though episodic, drinking. Moreover, Alkerwi et al [34] showed that "responsible alcohol intake" appears to be associated with a reduced prevalence of metabolic syndrome.
Favorable metabolic effect seemed to be restricted to alcohol consumption of less than $20 \mathrm{~g} /$ day among women, and of less than $40 \mathrm{~g} /$ day among men [34]. These findings support the actual recommendations regarding alcohol consumption among apparently healthy people, as reported by Alkerwi et al [34]. On the other hand, Thun et al [35] showed that, in a middle-aged and elderly population, moderate alcohol consumption slightly reduced overall mortality. The benefit depended, in part, on age and background cardiovascular risk and was far smaller than the large increase in risk produced by tobacco [35].

Furthermore, as reported by Sozio et al [36], a modest wine drinking might not confer sufficient benefits in cardiovascular or lipid profile in patients with obesity to justify any potential risk of worsening the underlying liver disease. It remains unclear, as showed by Sozio et al [36], why some obese patients with NAFLD have only steatosis whereas others exhibit steatohepatitis, and alcohol consumption could have a different effect in these two conditions.

Cotrim et al [37] showed that light-to-moderate alcohol consumption may have a protective effect against insulin resistance in severely obese patients. However, it had no impact on the severity of activity and stage of liver disease [37]. Furthermore, moderate alcohol consumption appears to protect against NAFLD as reported by Moryia et al [38]. Moreover, alcohol consumption was inversely associated with insulin resistance, independent of central obesity, metabolic profiles, and fatty liver diseases [39]. Hiramine et al [40] showed that alcohol consumption plays a protective role against fatty liver in men, and consistent alcohol consumption may contribute to this favorable effect. A cross-sectional study in Japanese men and women showed that light to moderate alcohol consumption has a favorable effect for fatty liver, but not for metabolic syndrome [41].

Baker et al [42] reported that the hyperactivity of all the genes regulating alcohol catabolism suggests that $j$ ) alcoholemia in NASH patients is elevated; jj) NASH patients show a particular tendency of the liver to reduce the circulating alcohol concentration. This evidence in humans lends credence to the hypothesis that alcohol may induce NAFLD [42].

Therefore, in addition to insulin resistance, alcohol may play a crucial pathogenic role in the development of NAFLD and its progression to NASH; for example, in alcohol drinkers who already have NAFLD and/or NASH, alcohol may cause synergistic injury, and in nondrinkers, obesity-related intestinal bacterial overgrowth may produce endogenous alcohol. It remains unclear why certain patients who are at risk for NAFLD are apparently able to derive the protective effects of alcohol, whereas those who already have NAFLD suffer worsening injury from alcohol.

\section{NEW RECREATIONAL DRUGS: A MAJOR HEALTH HAZARD}

Actually, numerous new drugs have materialized onto the recreational drugs market in Europe [43, 44]; ketamine and $\gamma$-hydroxybutyrate (GHB) are examples of these substances that have legitimate medical purposes. The aforementioned molecules have been largely substituted by many novel 
psychoactive products, which, due to their availability - in fact there is a florid online marketing - provoke politicians and health providers to initiate new strategies [45].

These new drugs are produced by chemical synthesis, i.e., 4-methylmethcathinone or mephedrone or extraction from traditional herbs, e.g., salvia divinorum and kratom [45]. The manufactured molecules are surreptitiously entered into the market to bypass the rigid laws concerning the violation of intellectual property rights of drugs and the protection of consumers, particularly avoiding the free flow of truthful information in the marketplace [45].

Actually, although mephedrone, belonging to the family of cathinones, and some synthetic molecules (cannabinoids) are classified as illegal drugs, the major part of these new psychoactive substances remain legal on the market [45]. Furthermore, unexpected changes in legislation, associated with rapid branding diversification as well as poor quality control, challenge buyers and, obviously, physicians to identify exactly what an addict consumes [45].

Despite the use of alcohol and tobacco, in a large sense considered two legally available recreational drugs, is increasing in the Western World, many data show adverse effects on organ function. Moreover, the use of illegal recreational drugs has reached epidemic proportions in many so-called civilized countries. It is evaluated that one out of four people in Western society has used recreational drugs at some times during his/ her life, usually in the younger age with pairs. It should be emphasized that the market is so florid that body packing and internal concealment, used by drug dealers to smuggle illicit substances, put the "body packer" at risk of gastrointestinal obstruction, creating a novel disease.

It is therefore inevitable that many physicians should manage and treat the dangerous effects associated with the abuse of these drugs.

\section{RECREATIONAL DRUGS AND THEIR HEPATOTOXICITY}

Hepatotoxicity due to Ecstasy (methylenedioxy methamfetamine, MDMA) is rare and can range from mild, self-limited episodes of liver damage to fulminant hepatitis requiring liver transplantation $[46,47]$. Some cases seem to be correlated to necrosis, secondary to hyperthermia. Others appear to result from a direct action of MDMA on hepatocytes. Several patients exhibit progressive jaundice and weight loss that could recur if exposed to MDMA again. Some other patients exhibit the disorder in acute crisis within hours from MDMA ingestion. Treatment should be tailored personally and ranges from nutritional support and careful longitudinal assessment of liver function to liver biopsy and transplantation.

MDMA is a significant cause of drug-induced liver failure [48]. This has two distinct features: the first associated with hyperpyrexia and the second presenting isolate. The former is evidenced by histology as a centrilobular necrosis and microvascular steatosis (as in heatstroke); the latter is quite always an acute cholestatic hepatitis with marked presence of eosinophils and histiocytes suggestive of a hypersensitivity reaction, as reported by Devlin et al [49]. The severity of these forms is clinically relevant, but unpredictable, in the sense that the onset is that of an acute hepatitis, sometimes rapidly evolving towards encephalopathy [49]. Unfortunately, there are no therapeutical options but the supportive one [49]. Inducing these addicts to abstain is of paramount importance because the re-exposure to the drug may provoke recurrence [49].

Cocaine is an alkaloid which is usually administered intra-nasally, intravenously or by inhalation. It is recognized as one of the most dangerous illicit recreational drugs [50, 51]. Hepatotoxicity evolves into a cytolytic hepatitis with an increase, generally significant, of the ALT levels a few hours after taking the dose, which can be partnered with jaundice [50]. It can be accompanied by shock, hypotension, hypoxia and high fever. An important point is that the cocaine-induced liver damage can be associated with rhabdomyolysis and subsequently acute renal failure. One possibility is that liver failure occurs in severe cases. An alternative explanation could be the presence of some metabolites responsible for hepatitis and other complications [50,51].

Buprenorphine is widely used for opiate withdrawal. Used orally, the risk of hepatotoxicity is rare [52]. On the other hand, when the product is used intravenously, the occurrence of cytolytic hepatitis is frequent, sometimes serious and fatal [52]. The difference in toxicity between the two routes of administration can be explained by the fact that intravenously the product reaches much higher liver concentrations that determine significant mitochondrial toxicity [52]. Toxicity may be increased if there is a simultaneous intake of another agent acting on mitochondria, as noted recently in patients using aspirin [52]. Finally, medicinal plants can be used for recreational scopes such as Kava Kava. This plant comes from the Islands of the South Pacific. Kava Kava originally had a ceremonial use due to its slightly euphoric and anxiolytic properties, obviously assumed in small doses. It is used in Western countries as an exhilarating substance and can induce severe cytolytic hepatitis, sometimes requiring liver transplantation, often resulting in death [53]. The sale of Kava Kava is prohibited on the markets of herbalism.

\section{HCV INFECTION AND CANNABIS}

Hepatic steatosis is frequently observed during chronic hepatitis C. It is either virus-induced, mostly related to $\mathrm{HCV}$ genotype 3, or environmental, mainly related to the consumption of alcohol or metabolic risk factors. Experimental work suggested a cannabinoid 1 (CB1) steatogenic effect. Indeed, it has been shown that endogenous hepatocytes CB1 receptor activation enhanced the synthesis of fatty acids and liver steatogenesis [54]. The role of CB1 and CB2 was evaluated in two experimental models of metabolic steatosis in mice. The administration of an antagonist of CB1 prevented the development of fatty liver and improved parameters of the metabolic syndrome [55]. In another study, CB2 contributed to inflammation associated with obesity, insulin resistance and steatosis with non-alcoholic origin [56]. These results demonstrate that $\mathrm{CB} 1$ and $\mathrm{CB} 2$ receptors have steatogenic properties.

The impact of recent (six months) use of cannabis (tetrahydrocannabinol) on the severity of steatosis was studied in 315 patients with HCV-related chronic hepatitis, who had 
never been treated. In multivariate analysis, daily cannabis use was an independent predictive factor associated with the presence of $\mathrm{j}$ ) severe steatosis (> $30 \%$ of hepatocytes), $\mathrm{jj}$ ) histological activity moderate to severe (A2 - A3, according to Metavir), HCV genotype 3, jjj) body mass index greater than or equal to $27 \mathrm{~kg} / \mathrm{m}^{2}$, jijj) hyperglycemia or diabetes, jijjj) the level of HCV RNA [57]. Conclusively, daily cannabis use is a liverrisk factor because it can accelerate the progression of fibrosis and worsen the severity of steatosis in patients with chronic hepatitis $\mathrm{C}$. These results are consistent with the experimental data that indicate pro-fibrogenetic properties of $\mathrm{CB} 1$ receptor and steatogenic capabilities of receptors CB1 and CB2.

As practical advice, physicians must search in the patients' history to discover whether a cannabis use is present when chronic hepatitis $\mathrm{C}$ has been diagnosed. It is much more frequent than is thought, being often hidden. The clinician must recommend untreated patients to ban the daily use of cannabis due to the risk of rapid fibrosis and progression of severe steatosis among daily smokers.

\section{ALCOHOL AND DIET RICH OF LIPIDS IN HCV INFECTION}

Purohit et al [58] showed that steatogenic substances such as ethanol, when coupled with a high-fat diet induce up-regulation of CB1 receptors by enhancing the synthesis of endocannabinoids, 2-arachidonoylglycerol and anandamide. These receptors are the same that are up-regulated in obese patients, stimulating on the one hand both lipogenic transcription factor sterol regulatory element-binding protein 1c (SREBP1c) with its target enzymes acetyl-CoA carboxylase-1 and fatty acid synthase, and on the other hand inhibiting carnitine palmitoyltransferase-1 [58]. As a consequence, an increased de novo fatty acid synthesis as well as reduced fatty acid oxidation leads to the well-known fatty liver, as a result. Hyperlipidic diet seems to activate CB2 receptors that further contribute to determining fatty liver and worsening the severity of fatty liver in hepatitis C patients [58].

\section{COMBINED USE OF RECREATIONAL DRUGS AND ALCOHOL}

Ketamine is one of the most widespread recreational drugs, regularly used in the so-called rave parties. Consumers frequently take it with alcoholic beverages. Alcohol ingestion in chronic or episodic use activates drug-metabolizing enzymes, thus decreasing the drugs' availability and reducing its effects. After these enzymes have been activated, they remain so even in the absence of alcohol drinking. For these reasons, recreational drugs users are compelled to increase the dose.

There is no evidence in humans, but animal models could help evaluate the toxicity. In a recent study by Way et al, ICR mice were treated for periods of 6,16 and 28 weeks with $30 \mathrm{mg} /$ $\mathrm{kg}$ ketamine injected daily intra-peritoneally and contextually with alcohol ( $0.5 \mathrm{ml}$ of $10 \%$ alcohol for each mouse) during the last 4 weeks of the treatment periods. This experiment ended up in a significant damage to the liver, including fatty degeneration of liver cells, fibrosis and increase in liver glutamic oxaloacetic transaminase, proliferative cell nuclear antigen and lactate dehydrogenase after 16 weeks of treatment with ketamine. The liver injury of these mice was particularly severe when the animals were treated with both ketamine and alcohol [59]. Finally, it is necessary to evidence that patients suffering from $\mathrm{HCV}$ infection, mainly the obese ones, due to their protracted status of depression [60], are natural candidates to the misuse of recreational drugs, including alcoholic beverages and cannabis, to try to better cope with a reduced quality of life and the burden of fibromyalgia. This latter disease is surprisingly improved by tetrahydrocannabinol [61].

\section{HEALTH POLICY}

Recreational drugs' use is incredibly common around the world and it very often leads to disaster and crime. To try to resolve this, some people advocate a liberal approach to legalization, while others support a strong government, policedriven "war" on the sale and use of drugs.

Even though literature data on effects of these recreational substances on hepatic steatosis are few and incomplete, some of them are reckoned as responsible for acute cytolytic hepatitis. The diagnosis of this illness should be primarily suspected in the less mature subjects. Sometimes the accountability of a recreational substance remains difficult to highlight since taking the product is often hidden by the user.

\section{FIVE-YEAR VIEW}

Alcohol and recreational drugs is a very hot topic, being not only a habit of the young, but impregnating broad sectors of the population, with many contrasting approaches and paradoxically a lack of data for other aspects. Recreational drugs are managed as a fun topic by scientists but the authors do not concur with what these scholars advocate. When not disregarded, recreational drugs are taken into consideration to inspire new medications as the case of tetrahydrocannabinol. This field will evolve in the future.

\section{CONCLUSIONS}

Evidence is in favor of the fact that alcohol and cannabis are factors that cause liver damage. Despite the effect of light alcohol consumption on decreasing insulin resistance and cardiovascular mortality, there does not seem to be a "safe" limit for alcohol consumption in the setting of pre-existing chronic liver disease. A deleterious role of daily use of recreational drugs, in particularly cannabis, has clearly been shown on the speed of progression of fibrosis and steatosis in patients suffering from chronic hepatitis C. Other recreational drugs, the consumption of which is increasing in Western countries, are potentially hepatotoxic, although not clearly inducing or worsening hepatic injury.

Conflicts of interest: There are no conflicts of interests regarding this review.

\section{REFERENCES}

1. Tarantino G. Non-alcoholic fatty liver disease, obesity and other illnesses. Clin Invest Med 2008;31:E290-E295. 
2. Fierbinteanu-Braticevici C, Negreanu L, Tarantino G. Is fatty liver always benign and should not consequently be treated? J Physiol Pharmacol 2013;64:3-9.

3. Colicchio P, Tarantino G, del Genio F, et al. Non-alcoholic fatty liver disease in young adult severely obese non-diabetic patients in South Italy. Ann Nutr Metab 2005;49:289-295.

4. Tarantino G, Colicchio P, Conca P, et al. Young adult obese subjects with and without insulin resistance: what is the role of chronic inflammation and how to weigh it non-invasively? J Inflamm (Lond) 2009;6:6.

5. Labruna G, Pasanisi F, Nardelli C, et al. UCP1 -3826 AG+GG genotypes, adiponectin, and leptin/adiponectin ratio in severe obesity. J Endocrinol Invest 2009;32:525-529.

6. Tarantino G, Pizza G, Colao A, et al. Hepatic steatosis in overweight/ obese females: new screening method for those at risk. World J Gastroenterol 2009;15:5693-5699.

7. Tarantino G, Colao A, Capone D, et al. Circulating levels of cytochrome $\mathrm{C}$, gamma-glutamyl transferase, triglycerides and unconjugated bilirubin in overweight/obese patients with non-alcoholic fatty liver disease. J Biol Regul Homeost Agents 2011;25:47-56.

8. Tarantino G, Finelli C, Colao A, et al. Are hepatic steatosis and carotid intima media thickness associated in obese patients with normal or slightly elevated gamma-glutamyl-transferase? J Transl Med 2012;10:50.

9. Finelli C, Tarantino G. Is visceral fat reduction necessary to favour metabolic changes in the liver? J Gastrointestin Liver Dis 2012;21:205208.

10. Finelli $C$, Tarantino $G$. Is there any consensus as to what diet or lifestyle approach is the right one for NAFLD patients? J Gastrointestin Liver Dis 2012;21:293-302.

11. Finelli C, Tarantino G. Have guidelines addressing physical activity been established in nonalcoholic fatty liver disease? World J Gastroenterol 2012;18:6790-6800.

12. Finelli $\mathrm{C}$, Tarantino $\mathrm{G}$. What is the role of adiponectin in obesity related non-alcoholic fatty liver disease? World J Gastroenterol 2013;19:802 812.

13. Tarantino G, Finelli C. What about non-alcoholic fatty liver disease as a new criterion to define metabolic syndrome? World J Gastroenterol 2013;19:3375-3384.

14. Tarantino G, Scalera A, Finelli C. Liver-spleen axis: Intersection between immunity, infections and metabolism. World J Gastroenterol 2013;19:3534-3542

15. Finelli C, Tarantino G. Should visceral fat, strictly linked to hepatic steatosis, be depleted to improve survival? Hepatol Int 2013;7:413-428.

16. Finelli C, Sommella L, Gioia S, La Sala N, Tarantino G. Should visceral fat be reduced to increase longevity? Ageing Res Rev 2013;12:996-1004.

17. Balkau B, Lange C, Vol S, Fumeron F, Bonnet F; Group Study D.E.S.I.R. Nine-year incident diabetes is predicted by fatty liver indices: the French D.E.S.I.R. study. BMC Gastroenterol 2010;10:56.

18. Woreta TA, Sutcliffe CG, Mehta SH, et al. Incidence and risk factors for steatosis progression in adults coinfected with HIV and hepatitis $\mathrm{C}$ virus. Gastroenterology 2011;140:809-817.

19. Potenza MN, Sofuoglu M, Carroll KM, Rounsaville BJ. Neuroscience of behavioral and pharmacological treatments for addictions. Neuron 2011;69:695-712.

20. Nestler EJ. Molecular mechanisms of drug addiction. Neuropharmacology 2004;47 Suppl 1:24-32.

21. Grueter BA, Rothwell PE, Malenka RC. Integrating synaptic plasticity and striatal circuit function in addiction. Curr Opin Neurobiol 2012;22:545-551.
22. Tan KR, Rudolph U, Lüscher C. Hooked on benzodiazepines: GABAA receptor subtypes and addiction. Trends Neurosci 2011;34:188-197.

23. Morales M, Pickel VM. Insights to drug addiction derived from ultrastructural views of the mesocorticolimbic system. Ann N Y Acad Sci 2012;1248:71-88.

24. Nielsen DA, Utrankar A, Reyes JA, Simons DD, Kosten TR. Epigenetics of drug abuse: predisposition or response. Pharmacogenomics 2012;13:1149-1160.

25. Robison AJ, Nestler EJ. Transcriptional and epigenetic mechanisms of addiction. Nat Rev Neurosci 2011;12:623-637.

26. Nestler EJ. Molecular basis of long-term plasticity underlying addiction. Nat Rev Neurosci 2001;2:119-128.

27. Tarantino G. Is Assessing the Presence of NASH by Liver Histology or Surrogate Markers Always Advisable? Hepat Mon 2013;13:e7560.

28. Caldwell SH, Lee VD, Kleiner DE, et al. NASH and cryptogenic cirrhosis: a histological analysis. Ann Hepatol 2009;8:346-352.

29. Arteel GE. Beyond reasonable doubt: who is the culprit in lipotoxicity in NAFLD/NASH? Hepatology 2012;55:2030-2032.

30. Chitturi S, Farrell GC. Etiopathogenesis of nonalcoholic steatohepatitis. Semin Liver Dis 2001;21:27-41.

31. Gäbele E, Dostert K, Dorn C, Patsenker E, Stickel F, Hellerbrand C. A new model of interactive effects of alcohol and high-fat diet on hepatic fibrosis. Alcohol Clin Exp Res 2011;35:1361-1367.

32. Wang Y, Seitz HK, Wang XD. Moderate alcohol consumption aggravates high-fat diet induced steatohepatitis in rats. Alcohol Clin Exp Res 2010;34:567-573.

33. Ekstedt M, Franzén LE, Holmqvist M, et al. Alcohol consumption is associated with progression of hepatic fibrosis in non-alcoholic fatty liver disease. Scand J Gastroenterol 2009;44:366-374.

34. Alkerwi A, Boutsen M, Vaillant M, et al. Alcohol consumption and the prevalence of metabolic syndrome: a meta-analysis of observational studies. Atherosclerosis 2009;204:624-635.

35. Thun MJ, Peto R, Lopez AD, et al. Alcohol consumption and mortality among middle-aged and elderly U.S. adults. N Engl J Med 1997;337:1705-1714.

36. Sozio MS, Chalasani N, Liangpunsakul S. What advice should be given to patients with NAFLD about the consumption of alcohol? Nat Clin Pract Gastroenterol Hepatol 2009;6:18-19.

37. Cotrim HP, Freitas LA, Alves E, Almeida A, May DS, Caldwell S. Effects of light-to-moderate alcohol consumption on steatosis and steatohepatitis in severely obese patients. Eur J Gastroenterol Hepatol 2009;21:969-972.

38. Moriya A, Iwasaki Y, Ohguchi S, et al. Alcohol consumption appears to protect against non-alcoholic fatty liver disease. Aliment Pharmacol Ther 2011;33:378-388.

39. Gunji T, Matsuhashi N, Sato H, et al. Alcohol consumption is inversely correlated with insulin resistance, independent of metabolic syndrome factors and fatty liver diseases. J Clin Gastroenterol 2011;45:808-813.

40. Hiramine $\mathrm{Y}$, Imamura $\mathrm{Y}$, Uto $\mathrm{H}$, et al. Alcohol drinking patterns and the risk of fatty liver in Japanese men. J Gastroenterol 2011;46:519-528.

41. Hamaguchi M, Kojima T, Ohbora A, Takeda N, Fukui M, Kato T. Protective effect of alcohol consumption for fatty liver but not metabolic syndrome. World J Gastroenterol 2012;18:156-167.

42. Baker SS, Baker RD, Liu W, Nowak NJ, Zhu L. Role of alcohol metabolism in non-alcoholic steatohepatitis. PLoS One 2010;5:e9570.

43. Hill SL, Thomas SH. Clinical toxicology of newer recreational drugs. Clin Toxicol (Phila) 2011;49:705-719. 
44. Hill SL, Doris T, Gurung S, et al. Severe clinical toxicity associated with analytically confirmed recreational use of 25I-NBOMe: case series. Clin Toxicol (Phila) 2013;51:487-492.

45. Winstock AR, Mitcheson L. New recreational drugs and the primary care approach to patients who use them. BMJ 2012;344:e288.

46. Henry JA, Jeffreys KJ, Dawling S. Toxicity and deaths from 3,4-methylenedioxymethamphetamine ("ecstasy"). Lancet 1992; 340:384-387.

47. Jones AL, Simpson KJ. Review article: mechanisms and management of hepatotoxicity in ecstasy (MDMA) and amphetamine intoxications. Aliment Pharmacol Ther 1999;13:129-133.

48. Andreu V, Mas A, Bruguera M, et al. Ecstasy: a common cause of severe acute hepatotoxicity. J Hepatol 1998;29:394-397.

49. Devlin RJ, Henry JA. Clinical review: Major consequences of illicit drug consumption. Crit Care 2008;12:202.

50. Mallat A, Dhumeaux D. Cocaine and the liver. J Hepatol 1991;12:275-278.

51. Van Thiel DH, Perper JA. Hepatotoxicity associated with cocaine abuse. Recent Dev Alcohol 1992;10:335-341.

52. Peyrière H, Tatem L, Bories C, Pageaux GP, Blayac JP, Larrey D. Hepatitis after intravenous injection of sublingual buprenorphine in acute hepatitis $\mathrm{C}$ carriers: report of two cases of disappearance of viral replication after acute hepatitis. Ann Pharmacother 2009;43:973-977.

53. Seeff LB. Herbal hepatotoxicity. Clin Liver Dis 2007;11:577-596.
54. Naughton SS, Mathai ML, Hryciw DH, McAinch AJ. Fatty Acid modulation of the endocannabinoid system and the effect on food intake and metabolism. Int J Endocrinol 2013;2013:361895.

55. Gary-Bobo M, Elachouri G, Gallas JF, et al. Rimonabant reduces obesityassociated hepatic steatosis and features of metabolic syndrome in obese Zucker fa/fa rats. Hepatology 2007;46:122-129.

56. Deveaux V, Cadoudal T, Ichigotani Y, et al. Cannabinoid CB2 receptor potentiates obesity-associated inflammation, insulin resistance and hepatic steatosis. PLoS One 2009;4:e5844.

57. Hézode C, Zafrani ES, Roudot-Thoraval F, et al. Daily cannabis use: a novel risk factor of steatosis severity in patients with chronic hepatitis C. Gastroenterology 2008;134:432-439.

58. Purohit V, Rapaka R, Shurtleff D. Role of cannabinoids in the development of fatty liver (steatosis). AAPS J 2010;12:233-237.

59. Wai MS, Chan WM, Zhang AQ, Wu Y, Yew DT. Long-term ketamine and ketamine plus alcohol treatments produced damages in liver and kidney. Hum Exp Toxicol 2012;31:877-886.

60. Tarantino G, Basile V, Conca P, et al. Could the depression of obese patients suffering from chronic hepatitis $\mathrm{C}$ be temporarily improved? J Viral Hepat 2008;15:646-650.

61. Fiz J, Durán M, Capellà D, Carbonell J, Farré M. Cannabis use in patients with fibromyalgia: effect on symptoms relief and health-related quality of life. PLoS One 2011;6:e18440. 